\title{
¿Hasta qué punto cumplen los estados miembro con la legislación de la Unión Europea? Teoría y factores empíricos sobre el proceso de implementación*
}

\author{
To what extent do Member states comply with EU legislation? Theories \\ and empirical factors about the implementation process
}

Javier Arregui

Jean Monnet Chair on EU Governance, Public Policy Center

Universitat Pompeu Fabra y Johns Hopkins University

doi: 10.18543/ced-55-2016pp165-191

Sumario: I. Introducción.-II. Teoría y factores explicativos del proceso de implementación.-III. Datos Empíricos sobre el nivel de conformidad de los EM con la legislación de la UE.-IV. ¿Existe en los EM un problema de conformidad con la legislación de la UE? - V. Conclusiones.

Resumen: El objetivo de este artículo de investigación es evaluar críticamente el estado del arte en relación al proceso de implementación legislativa de la Unión Europea y mostrar en qué medida existe un problema de deriva burocrática y de incumplimiento con la legislación europea por parte de los estados que conforman la Unión. Para conseguir dichos objetivos se plantean dos preguntas de investigación: ¿En qué medida los estados miembro cumplen con la legislación europea? y ¿Cuáles son los factores explicativos más relevantes que explican la falta de conformidad con la legislación europea? Para contestar dichas preguntas, el artículo utiliza una base de datos inédita que recoge todos los procedimientos de infracción oficiales que han tenido lugar en los estados miembro entre 1995 y 2015 . El estudio muestra que existe una variación muy alta entre estados en el nivel de conformidad y/o cumplimiento con la legislación europea. Esta variación se ha explicado de acuerdo a factores tales como la efectividad administrativa de los estados, el control parlamentario, la capacidad en los procesos de coordinación entre actores, y la existencia de posibles conflictos (de preferencias) entre estos actores que están implicados directamente en el proceso de implementación en cada uno de los estados analizados.

Palabras clave: conformidad legislativa, implementación, estados miembro, proceso político de la UE, infracciones.

Abstract: The aim of this research article is to critically assess the state of the art in relation to the process of legislative implementation of the European Un-

* Recibido el 19 de mayo de 2016, aceptado el 8 de julio de 2016. 
ion. This article also aims to show to what extent there is a problem of bureaucratic drift and failure to comply with European legislation by the states that make the Union. To reach these goals, two research questions are posed: To what extent member states comply with European legislation? and What are the most important explanatory factors behind the lack of conformity with European legislation? To answer these questions, the article uses a unique data base that collects all official infringement procedures that have taken place in member states between 1995 and 2015. The study shows that there is a very high variation between states in the degree of compliance with European legislation. This variation is explained according to factors such as the administrative effectiveness of a member state, parliamentary control, capacity in the coordination processes between actors, and the existence of potential conflicts (preferences) between these actors who are directly involved in the implementation process.

Keywords: legislative compliance, implementation, member states, EU policy process, infringements

\section{Introducción}

Los padres fundadores de los estudios sobre implementación legislativa, Jeffrey Pressman y Aaron Wildasvsky, escribieron en 1973 el libro Implementation: How Great Expectations in Washington are Dashed in Oakland. Su objetivo era explicar el desajuste entre las políticas elaboradas por Washington contra la pobreza y el poco éxito obtenido a la hora de reducir las bolsas de pobreza existentes. Estos autores señalan que el proceso de implementación legislativa es un proceso extraordinariamente complejo incluso en estas circunstancias en las que no tiene lugar un conflicto entre medios burocráticos y fines políticos. A partir de entonces, este tipo de estudios se han incorporado en mayor o menor medida al análisis sobre el proceso político en los diferentes sistemas políticos. Aunque los estudios sobre implementación se originaron dentro del contexto de los estados nacionales, en los últimos años se han desarrollado más allá de sus fronteras tradicionales. Más específicamente, en la Unión Europea (UE), tras el Tratado de Maastricht (una vez que oficialmente se completó la creación del mercado único en 1992) se empezaron a desarrollar investigaciones cuyo principal objetivo era el estudio sistemático sobre el grado de cumplimiento de la legislación europea en los estados miembro (EM) de la UE. Es a partir de este momento cuando este tipo de investigaciones empiezan a ocupar un papel cada vez más relevante en el estudio sobre el proceso político de la UE.

Pero, ¿Es importante el proceso de implementación? ¿Por qué se han de ocupar los politólogos sobre el proceso de implementación? La respuesta 
es sencilla: el hecho de que la UE adopte decisiones políticas en forma de leyes no significa que los EM adopten e implementen el contenido de estas leyes en el tiempo acordado y/o de un modo correcto. De hecho, existe una creciente literatura en Ciencia Política que muestra como en prácticamente todos los sistemas políticos del mundo existe una deriva burocrática, que confirma el hecho de que con demasiada frecuencia existen diferencias substantivas entre lo que dice una ley y la política que se aplica en realidad.

La UE no dispone de un sistema administrativo propio que pueda articular el proceso de implementación de la legislación europea en cada EM. En este sentido, el sistema político de la UE se caracteriza por tener una estructura de implementación bastante descentralizada. Mientras que el proceso de negociación y decisión tiene lugar en Bruselas, el proceso de implementación se lleva a cabo en cada uno de los 28 EM. Esto supone, de hecho, que existen 28 maneras diferentes de poner en práctica la legislación europea. Mientras que la existencia de diferentes tradiciones en el proceso de implementación legislativa no es en sí mismo un inconveniente, sí que se puede convertir en un problema cuando unos estados aplican la legislación y otros no. Esta disparidad puede llevar a un déficit en la implementación de la legislación europea.

En este sentido, el objetivo principal de la presente investigación es evaluar críticamente el estado del arte y de la literatura en relación al proceso de implementación legislativa europea en los diferentes EM y mostrar hasta qué punto existe un problema de deriva burocrática y de incumplimiento con la legislación europea. Para conseguir dichos objetivos, esta investigación plantea dos preguntas de investigación. Por un lado, ¿Hasta qué punto los diferentes EM de la UE cumplen de un modo sistemático con la legislación adoptada colectivamente en Bruselas? Por otro lado, se plantea una pregunta explicativa que complementa a la anterior: ¿Cuáles son los factores más relevantes que explican la falta de conformidad con la legislación europea?

Estas preguntas de investigación son relevantes en la medida en que la correcta implementación de una ley es un aspecto clave para el buen desarrollo del proceso de integración. En el caso de que una ley no se implemente de un modo correcto y/o simultáneo puede llegar a debilitar el funcionamiento del mercado interno. Ello puede producir consecuencias no deseadas para los principales actores económicos que participan en el proceso de integración: las empresas y los ciudadanos, lo cual podría socavar la eficacia y la eficiencia del mismo proceso de integración, y dependiendo de su intensidad podría llegar a poner en peligro incluso el mismo proceso de integración ${ }^{1}$.

${ }^{1}$ Markus Haverland, Bernard Steunenberg y Frans Van Waarden, «Sectors at Different Speeds: Analyzing Transpositions Deficits in the European Union», Journal of Common Market Studies, 49, n. 2 (2011): 265-291. 
El concepto de implementación política hace referencia a lo que pasa después de que una decisión vinculante se convierte en ley ${ }^{2,3}$. Por tanto, el proceso de implementación legislativa evalúa el proceso de convertir una política en una acción determinada, y tal y cómo nos recuerda Versluis ${ }^{4}$, la ley en los libros no es necesariamente lo mismo que la ley en la acción. Para que el proceso de implementación legislativa tenga un mínimo de eficacia es preciso proporcionar medios y mecanismos (fundamentalmente de naturaleza administrativa) que posibiliten el cumplimiento de la ley europea al mismo tiempo que se acredita poder detectar su incumplimiento.

El presente estudio cubre todo tipo de infracciones en la legislación vinculante para los EM, esto es, Directivas, Reglamentos, Decisiones y los artículos de los Tratados de la UE. El estudio muestra información sobre todos los procesos de infracción abiertos por la Comisión Europea y el Tribunal de Justicia de la Unión Europea (TJUE) en el periodo 1995-2015. Esto nos proporcionará información sistemática para identificar posibles problemas de naturaleza estructural. Además, esta investigación desagrega dicha información por áreas políticas, en el caso de España, para poder tener una instantánea más completa y substantiva sobre el proceso de implementación de la legislación europea en España.

La siguiente sección introduce de un modo sistemático el concepto de implementación legislativa en la UE, describe y explica las fases de dicho proceso así como los actores que están implicados de un modo directo en este proceso. La tercera sección muestra las principales teorías y variables explicativas que se han utilizado hasta la fecha para explicar los procesos de implementación legislativa en la UE. La cuarta sección muestra datos empíricos sobre los problemas de implementación de los diversos EM en el periodo 1995 y 2015 . La siguiente sección evalúa en qué medida existe un problema con el (in)cumplimiento de la legislación europea por parte de los EM. Finalmente, se elaboran las conclusiones de la investigación.

${ }^{2}$ Eugene Bardach, The Implementation Game: What happens after a bill becomes a law (Cambridge: MIT Press, 1977).

${ }^{3}$ Un concepto que está directamente relacionado con el proceso de implementación legislativa en la UE es el de Europeización. Este concepto se centra en evaluar los efectos que el proceso de integración europea produce en los EM. El proceso de Europeización origina cambios inequívocos en el nivel doméstico. Desde esta perspectiva, el proceso de implementación de los EM es uno de los mecanismos más importantes del proceso de Europeización: cuanto más efectiva sea la implementación de la legislación europea mayor será el impacto de Europeización dentro de un EM.

${ }^{4}$ Esther Versluis, «Explaining variations in implementation of EU Directives», European Integration online Papers, $n .^{\circ} 8$ (2004): 1-17. 


\section{Concepto, fases y actores del Proceso de Implementación en la UE}

La UE dispone de una diversidad importante de leyes. La tabla 1 muestra los diferentes tipos de leyes que se elaboran en la UE, los ámbitos que cubren así como el tipo de obligación que supone para los EM en el proceso de implementación legislativa. Tal y como esta tabla indica, los tres tipos de leyes más importantes son de obligada implementación. Las Directivas, a través de un proceso de transposición en la legislación nacional y los Reglamentos y las Decisiones de aplicación directa en los EM. Este tipo de leyes cubren las áreas políticas más relevantes para el funcionamiento del mercado interno, los derechos fundamentales y algunos aspectos de la política fiscal y macroeconómica. Mientras que el resto de leyes, son leyes blandas o acuerdos conseguidos en la UE con distintos niveles de obligatoriedad en su implementación. El alcance de estas normas va desde los acuerdos en política macroeconómica y vigilancia fiscal (que son de obligada ejecución) hasta los acuerdos adoptados a través del Método Abierto de Coordinación (sobre empleo, política social, etc) en las que la obligatoriedad a la hora de implementar dichas normas es mucho más laxa.

\section{Tabla 1}

Tipología de Leyes en la UE y su relación con el proceso de implementación en los estados miembros 5

\begin{tabular}{c|l|l}
\hline Tipo de ley & Tipo de implementación & \multicolumn{1}{c}{ Áreas políticas que cubren } \\
\hline \multirow{2}{*}{$\begin{array}{c}\text { Leyes vinculantes } \\
\text { (Directivas, } \\
\text { Reglamentos, } \\
\text { Decisiones) }\end{array}$} & $\begin{array}{l}\text { Implementación } \\
\text { obligatoria }\end{array}$ & $\begin{array}{l}\text { La maýa de políticas en la construc- } \\
\text { ción del mercado: mercado interno, agri- } \\
\text { cultura, comercio, industria, pesca, unión } \\
\text { monetaria, competición, política regional, } \\
\text { I+D, política social, medioambiente, con- } \\
\text { sumidores, desarrollo, JHA-AFSJ (desde } \\
\text { Ámsterdam) } \\
\text { Derechos fundamentales (desde Lis- } \\
\text { boa) } \\
\text { Algunos aspectos de vigilancia fiscal y } \\
\text { macroeconómica (desde las reformas del } \\
\text { año 2011). }\end{array}$ \\
\hline
\end{tabular}

5 Esta tabla es de elaboración propia con información proveniente de diversas fuentes de naturaleza legal que incluye tanto legislación secundaria como legislación proveniente de los Tratados. 


\begin{tabular}{|c|c|c|}
\hline Tipo de ley & Tipo de implementación & Áreas políticas que cubren \\
\hline \multirow{4}{*}{ Leyes Blandas } & $\begin{array}{l}\text { Obligación en la } \\
\text { implementación pero } \\
\text { ejecución laxa }\end{array}$ & $\begin{array}{l}\text { Vigilancia fiscal y macroeconómica } \\
\text { (antes de las reformas del año 2011). }\end{array}$ \\
\hline & $\begin{array}{l}\text { Obligación en la } \\
\text { implementación pero } \\
\text { sin ejecución }\end{array}$ & $\begin{array}{l}\text { Algunos aspectos de la Política Exte- } \\
\text { rior y de Seguridad. }\end{array}$ \\
\hline & $\begin{array}{l}\text { Obligación blanda/ } \\
\text { implementación dura }\end{array}$ & $\begin{array}{l}\text { Algunos aspectos de vigilancia fiscal y } \\
\text { macroeconómica (desde las reformas del } \\
\text { año 2011). }\end{array}$ \\
\hline & $\begin{array}{l}\text { Obligación blanda/ } \\
\text { implementación laxa }\end{array}$ & $\begin{array}{l}\text { Método abierto de coordinación: em- } \\
\text { pleo, coordinación económica, pensiones } \\
\text { e inclusión social. } \\
\text { Algunos aspectos sobre competición: } \\
\text { medioambiente, energía, transporte, cul- } \\
\text { tura y educación, política regional, indus- } \\
\text { tria, desarrollo e I+D }\end{array}$ \\
\hline
\end{tabular}

El proceso de implementación de la legislación europea abarca diferentes fases que incluye: 1) la transposición legal; 2) la implementación administrativa; y 3) la ejecución práctica de la ley. La transposición legal hace referencia al cómo y cuándo se incluye una Directiva europea (norma vinculante) en la legislación nacional. La implementación administrativa se refiere a los procesos de cambio en la introducción de las nuevas leyes y en qué medida las distintas administraciones y actores administrativos se implican en dicho proceso. La ejecución práctica de la ley hace referencia tanto a la manera en la que se implementa una ley como a la forma en la que se penaliza los incumplimientos de la misma.

Tan pronto como la Comisión tiene constancia de la existencia de una implementación legislativa incorrecta comienzan a activarse los mecanismos para hacer cumplir la ley europea. El artículo 226 del Tratado de la UE otorga a la Comisión Europea el derecho a iniciar un procedimiento de infracción contra los EM que incumplan con cualquiera de las obligaciones que se establecen en los Tratados de la UE. La Comisión puede abrir procedimientos de infracción de acuerdo con los siguientes motivos: 1) Cualquier violación de los artículos de un Tratado, de los Reglamentos o de las Decisiones, todos ellos de aplicación directa por parte de los EM; 2) La notransposición de una Directiva (o el retraso en su transposición) a la legislación nacional; 3) Una incorrecta transposición de una Directiva a la legislación doméstica; 4) La no (o incorrecta) implementación práctica de una 
Directiva (una vez que ya ha sido transpuesta) o de cualquier otra ley de naturaleza vinculante; 5) El incumplimiento de las decisiones del TJUE ${ }^{6}$.

Existen tres mecanismos que la Comisión Europea puede utilizar para abrir un procedimiento de infracción a un EM:

- Carta de Notificación. En una primera fase, si la Comisión identifica alguna violación de una ley europea, envía una primera carta de notificación que informa al EM correspondiente sobre la violación de dicha ley. También le insta a adoptar las medidas necesarias para que se adecúe lo antes posible a la ley europea. El propósito de esta notificación motivada es asegurarse de que se ha informado al EM con el detalle necesario sobre los motivos que le han llevado a abrir un primer expediente de infracción. La Comisión tiene la obligación de especificar con claridad los principales puntos de controversia. Esto es un punto fundamental, para que, posteriormente, cada EM pueda defender sus intereses de un modo eficaz. Tras esta primera notificación, cada EM tiene un tiempo para introducir cambios así como para comunicar la aplicación de dichos cambios a la Comisión.

- Dictamen Motivado (reasoned opinion). En esta segunda fase, la Comisión ha de proporcionar una exposición coherente y detallada de las razones que han llevado a la conclusión de que se ha producido un incumplimiento de una ley. En esta fase se examina minuciosamente la respuesta dada por cada EM a los requerimientos de la carta de notificación. Si esta respuesta no es convincente para la Comisión, esta llevará el caso ante la CEJ.

- Caso ante el TJUE. Una vez que el EM persiste en la no conformidad con la legislación europea, el TJUE deberá emitir un veredicto final que podría incluir una penalización administrativa y/o económica $^{7}$. El TJUE sólo puede dictar una sentencia en relación a los puntos controvertidos que se han dado a conocer al EM en la carta de notificación y/o en el dictamen motivado. Por otra parte, las po-

${ }^{6}$ Al mismo tiempo, los gobiernos que no cumplen con la legislación europea se exponen a que puedan iniciarse procedimientos legales adicionales por parte del TJUE auspiciados por actores domésticos que tengan un interés especial en que se lleve a cabo una determinada modificación legislativa que vaya en la línea de la doctrina del TJUE. Es un mecanismo que Blauberger (2014) ha bautizado como obediencia anticipada. Para evitar este tipo de procesos añadidos, algunos EM deciden seguir la doctrina del TJUE incluso en circunstancias en las que (legalmente) podrían quedar al margen de seguir dicha doctrina (ver Obermaier 2008).

7 Sin embargo, no existe una fuerza judicial de la UE que obligue a los estados a acatar las reglas europeas. En este sentido, el marco legal europeo y su capacidad más o menos limitada se situaría en un espacio intermedio entre la legislación doméstica y las normas de naturaleza internacional. 
sibles sanciones impuestas por el TJUE deben basarse en los puntos sobre los que cada EM ha ejercido su defensa.

Hasta la fecha ha existido en la literatura una tendencia a centrar el análisis en las fases iniciales del proceso de implementación. Esto es debido a que existe un mayor número de procedimientos abiertos en las fases iniciales (básicamente, cartas de notificación que alertan sobre problemas en el proceso de transposición) y, por tanto, mayor número de casos para explicar dicha variación. De acuerdo con esta lógica, una mayoría de estudios empíricos han adoptado como variable dependiente el proceso de transposición ${ }^{8}$. Un menor número de estudios se han centrado en los procedimientos de infracción abiertos por el TJUE ${ }^{9}$. Estos últimos estudios incluyen información no sólo sobre el proceso de transposición sino también sobre la implementación administrativa así como sobre la implementación real de la ley. Por tanto, proporcionan una información más completa que los estudios que se centran únicamente en el proceso de transposición.

Aunque cada EM organiza el proceso de implementación de una manera individualizada, existen algunas similitudes entre EM. Por ejemplo, la implementación legal (la transposición de Directivas) se suele gestionar desde el ministerio nacional responsable del tema que ha de implementarse. La situación es más compleja en los estados federales y/o altamente descentralizados, donde los ministerios de los estados federados también pueden estar implicados directamente en el proceso de transposición, sobre todo si son responsables, total o parcialmente, del área de la ley que se ha de transponer. Por ejemplo, en Alemania, España o Bélgica, los estados federados o las Comunidades Autónomas (CCAA) pueden tener un papel importante en la transposición a la legislación doméstica de algunos temas. Además, por ejemplo, en el caso de España, las CCAA son los actores responsables de la implementación práctica de toda la legislación europea.

Dentro del ámbito doméstico, existe toda una constelación de actores que pueden desempeñar un papel relevante en el proceso de implementación de las leyes europeas. Estos pueden ir desde actores muy politizados (actores gubernamentales y grupos de intereses) hasta actores con un perfil de naturaleza más técnica y burocrática. El tipo de ley (Directiva, Reglamento o Decisión) a implementar es el que determina qué tipo de ac-

8 Dimiter Toshkov, «Taking Stock: A review of quantitative studies of transposition and Implementation of EU Law», EIF Working Paper, n. ${ }^{\circ} 1$ (2010): 1-44.

9 Tanja A. Börzel et al., «Obstinate and Inefficient: Why Member States do not comply with European law», Comparative Political Studies, 43, n. 11 (2010): 1363-1390; Marco Guilani, «Europeanization in Comparative perspective: Institutional Fit and National Adaptation», en The Politics of Europeanization, ed. por Kevin Featherstone y Claudio M. Radaelli (Oxford: Oxford University Press, 2003), 134-157. 
tores intervienen en mayor o menor medida. Por ejemplo, una Directiva al tener que transponerse en la legislación doméstica hace que los actores políticos sean más relevantes ya que han de elaborarse decretos, órdenes ministeriales o leyes domésticas (en algunos casos) para poder transponer dicha Directiva. Además, aquí también existe variación entre EM. Mientras que en algunos estados es suficiente una orden ministerial para transponer una directiva en otros es el parlamento nacional quien ha de intervenir directamente. Sin embargo, en la mayoría de los casos la transposición correcta de las Directivas depende fundamentalmente de las capacidades administrativas y de la sofisticación en los procesos de coordinación entre diversos actores, así como en la experiencia de los mismos en este tipo de procesos ${ }^{10}$. La variación sustantiva que existe entre los EM en el proceso de transposición (en cuanto a requerimientos legales, actores que intervienen, tipos de coordinación, capacidades, etc.) puede determinar de un modo decisivo el desempeño de los distintos estados en el proceso de implementación.

\section{Teoría y factores explicativos del proceso de implementación}

Las preocupaciones sobre el destino de la legislación europea en los EM comenzaron en una fecha tan temprana como los años $80^{11}$. Estos estudios, con un enfoque netamente legal, son los pioneros sobre el proceso de implementación de la legislación europea en los EM. Concebían este más como un proceso administrativo que como un proceso político, donde los aspectos procedimentales y administrativos adquirían un papel central. Posteriormente, una vez concluida la construcción del mercado en 1992 y comenzado el proceso de europeización de las políticas nacionales, el centro de atención se empezó a poner en el grado de compatibilidad entre las políticas de la UE y las estructuras de gobierno domésticas. Es a partir de este momento cuando se empiezan a identificar los EM con una estructura de gobernanza doméstica más o menos eficaz en relación a la implementación de la legislación europea. También es a partir de este momento, cuando se exponen por primera vez los problemas de agencia, donde los gobiernos nacionales, regionales o las administraciones son vistas como «guar-

10 Oliver Treib, «Implementing and Complying with EU Governance Outputs», Living Review in European Governance, 9, n. ${ }^{\circ} 1$ (2014): 1-47.

${ }^{11}$ Guiseppe Ciavarini Azzi, L'application du droit communautaire par les etats membres (Maastricht: European Institute of Public Administrations, 1985); Heinrich Siedentopf y Jacques Ziller, Making European Policies Work: The Implementation of Community Legislation in the Member States (London: Sage, 1988). 
dianes del status quo ... que protegen las tradiciones legal-administrativas nacionales» ${ }^{12}$.

La mayor parte de los estudios sobre implementación utilizan dos enfoques generales: por un lado, el enfoque top-down, que concibe el proceso de implementación como una aplicación de la ley con una intención política manifiesta que se define en el centro; por otro, el enfoque bottom-up argumenta que las políticas se configuran de un modo decisivo en las estrategias que llevan a cabo las redes de actores que aplican la legislación en el mundo real ${ }^{13}$.

Recientemente los estudios sobre implementación en la UE han comenzado a utilizar una pluralidad de enfoques teóricos y analíticos diversos, que desde una perspectiva general se fundamentan tanto en los enfoques top-down como en los enfoques bottom-up. Las teorías que se han utilizado hasta la fecha con más intensidad para explicar la variación en el proceso de implementación legislativa en los EM se reducen principalmente a dos: una primera que se centra en la gestión y una segunda que pone especial atención en la ejecución. La escuela de gestión argumenta que los principales factores que explican el proceso de implementación están relacionados con una diversidad de factores relacionados con la calidad administrativa, el nivel de desajuste entre la nueva ley y el status quo, las prácticas reales o factores culturales. Por otro lado, los teóricos de la ejecución argumentan que habitualmente existe un problema de acción colectiva en los procesos de implementación legislativa y, por tanto, el peligro de free-riding está siempre presente en este tipo de procesos.

Hay dos teorías adicionales que también han sido utilizadas en la literatura de la UE, aunque con mucha menos intensidad: el enfoque constructivista y el enfoque normativo. El enfoque constructivista entiende el incumplimiento de la legislación europea como una carencia por parte de diversos actores a la hora internalizar las normas de la UE. De acuerdo con este tipo de razonamiento, cuanto más tiempo un EM ha formado parte de la UE, mayor tiempo habrá estado expuesto a su sistema normativo, y por tanto, mayor probabilidad de que dicho EM internalice dichas normas ${ }^{14}$. Desde el enfoque normativo se sostiene que el cumplimiento con la legalidad vigente

12 Francesco G. Duina, «Explaining Legal Implementation in the European Union», International Journal of the Sociology of law, 25 (1997): 155-179.

13 Paul A. Sabatier e Daniel A. Mazmanian, «The Implementation of Public Policy: A Framework of Analysis», en Effective Policy Implementation, ed. por Paul A. Sabatier e Daniel A. Mazmanian (Lexington: Lexington Books, 1981).

14 Abram Chayes, Antonia Handler Chayes y Ronald B. Mitchell, «Managing Compliance: A Comparative Perspective», en Engaging Countries: Strengthening Compliance with International Environmental Accords, ed. por Edith Brown Weiss e Harold Jacobson (Cambridge: MIT press, 1998), 39-62. 
puede tener por sí mismo suficiente fuerza para que los EM cumplan de un modo efectivo con la legislación europea y si existen casos en que no es así, este ha de combinarse siempre con otro tipo de variables, pero los aspectos normativos han de ser centrales en cualquier tipo de análisis ${ }^{15}$.

Las teorías sobre la ejecución legislativa están relacionadas con las teorías de la elección racional. Desde esta enfoque, el cumplimiento de la legislación no deja de ser un mecanismo intencional de los EM, que han de adoptar una decisión racional a la hora de cumplir (o no cumplir) con una ley, todo ello basado en el análisis coste/beneficio. De este modo, cuando un actor incumple una ley, no deja de ser una consecuencia del coste que tiene el cumplimiento de dicha ley para ese actor. Un actor o grupo de actores podría valorar que implementar una ley tiene un coste demasiado elevado. En consecuencia, este enfoque argumenta que los actores sólo cumplirán con la legislación europea cuando existan sanciones efectivas y significativas ${ }^{16}$. Si este último argumento es cierto, los estados sólo cumplirán con la legislación europea si existe un sistema efectivo para detectar las violaciones e infracciones de los EM, así como un sistema de sanciones lo suficientemente contundente como para evitar posibles tentaciones de incumplir con la legislación europea.

Desde esta misma teoría también se ha argumentado que el interés de un EM en el largo plazo sería cumplir con la legislación europea ya que los beneficios para los gobiernos podrían ser superiores. En este sentido, Garret ${ }^{17}$, por ejemplo, sostiene que mientras que los incentivos en el corto plazo para incumplir con la legislación europea pueden ser altos, los EM pueden llegar a percibir racionalmente que los beneficios de una UE que funcione y salvaguarde el mercado interior pueden ser mayores en el largo plazo.

Además, es preciso tener presente que siguiendo el argumento de Keohane y $\mathrm{Nye}^{18}$, sobre poder e interdependencia entre estados, algunos estados son más sensibles (que otros) a la reputación y a los costes materiales impuestos por terceros actores. Ello puede condicionar su desempeño en

15 Derek Beach, «Why Governments Comply: An Integrative Compliance Model that Bridges the Gap Between Instrumental and Normative Models of Compliance», Journal of European Public Policy, 12, n. 1 (2005): 113-142.

16 Jonas Tallberg, «Paths to Compliance: Enforcement, Management and the European Union», International Organization, 56, n. 3 (2002): 609-643; George W. Downs, David M. Rocke y Peter N. Barsoom, «Is the Good News about Compliance also Good News about Cooperation?», International Organization, 50, n. 3 (1996): 379-406.

17 Geoffrey Garret, «The Politics of legal integration in the European Union», International Organization, 49, n. ${ }^{\circ} 1$ (1995): 171-181.

18 Robert Keohane y Joseph Nye, Power and interdependence (Boston: Little Brown and Company, 1977). 
el proceso de implementación de la legislación europea. Tal y como señala Börzel $^{19}$, los estados más pequeños tienen menos poder político y económico y son más dependientes de terceros actores para una mayor cooperación. En este sentido, los EM menos poderosos serían más sensibles a constreñimientos externos impuestos por sanciones y, por tanto, menos dados a infringir la legislación europea ${ }^{20}$. Por otro lado, los estados con más poder y peso político, incluso si su reputación disminuye como consecuencia del incumplimiento de la legislación, su poder político y económico les salvaguarda a la hora de influir en la $\mathrm{UE}^{21}$.

Las teorías del management (gestión) argumentan que es la incapacidad más que la falta de voluntad lo que está detrás del incumplimiento de los estados con la legislación europea ${ }^{22}$. El argumento central que utilizan es que en líneas generales los EM están dispuestos a cumplir con las leyes internacionales y cuando tiene lugar un incumplimiento se puede explicar como resultado, o bien de la incapacidad de un estado, o bien de la ambigüedad de la norma. Sin embargo, el concepto de capacidad de un EM no se ha utilizado de un modo uniforme en la literatura. Cuando se habla de capacidades de los EM, este enfoque se refiere, habitualmente, a las capacidades financieras, tecnológicas, científicas o administrativas de dichos estados. Además, desde esta teoría también se ha argumentado que las estructuras institucionales de un país pueden limitar su capacidad e influen$\mathrm{cia}^{23}$. Por ejemplo, la cantidad y cualidad de jugadores veto que existen dentro de un sistema político puede condicionar de un modo relevante el proceso de implementación de la legislación europea ${ }^{24}$. Los EM con mayor número de jugadores veto disponen de menos capacidad para cambiar el status quo ${ }^{25}$. Por tanto, la autonomía de un gobierno puede estar relacionada con el número de jugadores veto que existen en un país mientras que la capacidad de ese mismo gobierno puede estar más relacionada con los recursos humanos y financieros de dicho estado. Ambos tipos de fac-

19 Tanja A. Börzel, «Non-compliance in the European Union: pathology or statistical artefact», Journal of European Public Policy, 8, n. 5 (2001): 803-824.

${ }^{20}$ Lisa L. Martin, Coercive Cooperation: Explaining Multilateral Economic Sanctions (Princeton: Princeton University Press, 1992).

${ }^{21}$ Robert Thomson et al, The European Union Decides: Testing theories of European Decision Making (Cambridge: Cambridge University Press, 2006).

${ }^{22}$ Chayes, «Managing Compliance: A Comparative Perspective»

23 Peter Evans, Embedded Autonomy: States and industrial Transformations, (Princeton: Princeton University Press, 1995).

${ }^{24}$ Markus Haverland, «National Adaptation to European Integration: The Importance of institutional Veto Points», Journal of Public Policy, 20, n. 1 (2000): 83-103.

${ }^{25}$ George Tsebelis, Veto Players: How Political Institutions Work (Princeton: Princeton University Press, 2002). 
tores son relevantes ${ }^{26}$ y están directamente relacionados con la eficacia de la burocracia que tiene cada EM a la hora implementar la legislación europea.

La literatura ha utilizado las mismas variables para explicar cualquier problema en el proceso de implementación, independientemente de que el objeto de estudio hayan sido los retrasos o problemas en el proceso de transposición o el examen de las infracciones abiertas por el TJUE. Tal y como argumentan Toshkov ${ }^{27}$ y Treib $^{28}$ no existen variables explicativas únicas para cada una de las fases. Dicho de otra forma, la mayoría de estudios se han comportado como si los problemas de implementación siguieran una lógica teórica unitaria (cuando obviamente no es así), ya que diversos factores pueden influir y de hecho influyen en distintas fases del proceso. Esto es así, entre otras razones, porque en las distintas fases del proceso de implementación intervienen distintos actores.

Las variables más utilizadas, hasta la fecha, relacionadas con el enfoque de gestión hacen referencia, en primer lugar, a la eficacia administrativa. Esta es una variable recurrente en los procesos de implementación legislativa. Con frecuencia se argumenta que el proceso de implementación de la UE depende, en gran medida, de la eficacia de la burocracia doméstica que ha de implementar la legislación ${ }^{29}$. La capacidad de una burocracia se entiende, con frecuencia, como una variable distinta a su eficacia ${ }^{30}$. En este sentido, la capacidad de una burocracia puede incluir variables como la cantidad de directivas que un EM llega a transponer durante un año. Los factores culturales de un EM en sentido amplio también pueden llegar a ser una variable relevante. Por ejemplo, Berglund ${ }^{31}$ argumenta que hay EM en los que el estado de derecho funciona mejor que en otros. Per-

${ }^{26}$ Heather A. D. Mbaye, «Why National States Comply with Supranational Law Explaining Implementation Infringements in the European Union 1971-1993», European Union Politics, 2 , n. ${ }^{\circ} 3$ (2001): 259-281.

27 Toshkov, « Taking Stock: A review of quantitative studies of transposition and Implementation of EU Law»

28 Treib, «Implementing and Complying with EU Governance Outputs»

${ }^{29}$ Michael Kaeding, «Determinants of Transpostition delay in The European Union», Journal of Public Policy, 26, n. 3 (2006): 229-253; Haverland, Steunenberg y Van Waarden, «Sectors at Different Speeds: Analyzing Transpositions Deficits in the European Union»

${ }^{30}$ Duina, «Explaining Legal Implementation in the European Union»; Tanja A. Börzel, «Participation Through Law Enforcement: The Case of the European Union», Comparative Political Studies, 39, n. ${ }^{\circ} 1$ (2006): 128-152; Mariyana Angelova, Tanja Dannwolf y Thomas König, «How Robust are Compliance Findings? A Research Synhtesis», 19, n. . 8 (2012): 1269-1291.

31 Sara Berglund, Putting Politics into Perspective: A study on the Implementation of EU Public Utilities Directives (Delft: Derbur, 2009). 
kins y Neumayer ${ }^{32}$ añaden que también existen diferencias significativas entre los EM a la hora de resolver conflictos y/o de hacer aplicar el estado de derecho. Otro factor explicativo que se ha utilizado (dentro de esta escuela de gestión) es lo que se llama el poder y la capacidad de coordinación de los distintos actores ${ }^{33}$. El argumento central aquí es que cuanto mayor sea el número de actores que haya que coordinar (y por tanto jugadores veto) mayor será el potencial de bloquear acciones que lleven a una transposición e implementación correcta de las leyes. Dentro de este argumento entran distintos tipos de variables de naturaleza institucional que pueden llegar a adquirir una relevancia significativa: el número de jugadores con capacidad de vetar el proceso $^{34}$, la forma en la que se estructura la relación entre el legislativo y el ejecutivo ${ }^{35}$, la implicación de los parlamentos nacionales en el proceso de transposición e implementación ${ }^{36}$, la coordinación entre ministerios ${ }^{37} \mathrm{o}$ la dinamización del proceso legislativo ${ }^{38}$. La configuración de cada una de estas variables en los EM puede ser determinante. Una mayoría de estudios también señalan la complejidad de una directiva a la hora de explicar problemas en su implementación: cuanto más compleja es una directiva mayor dificultad a la hora de implementarla correctamente. Las medidas de complejidad utilizadas hasta la fecha son la longitud del texto y el tiempo asignado para la transposición ${ }^{39}$, o el grado de discrecionalidad ${ }^{40}$.

Las variables explicativas más utilizadas en el enfoque de ejecución están relacionadas con las preferencias de los actores que intervienen en cada

${ }^{32}$ Richard Perkins y Eric Neumayer, «Do membership benefits by regulatory compliance? An empirical analysis of EU Directives 1978-99», European Union Politics, 8, n. ${ }^{\text {o }} 2$ (2007): 180-206.

${ }^{33}$ Dimiter Toshkov, « Taking Stock: A review of quantitative studies of transposition and Implementation of EU Law» Angelova, Dannwolf y König, «How Robust are Compliance Findings? A Research Synhtesis»

34 Tsebelis, Veto Players: How Political Institutions Work

35 Scott Nicholas Siegel, The Political Economy of Noncompliance: Adjusting to the Single European Market (Routdledge: Abingdon, 2011).

${ }^{36}$ Katerina Linos, «How can international Organizations Shape National Welfare States? Evidence from Compliance with European Union directives», Comparative Political Studies, 40 n. ${ }^{\circ} 5$ (2007): 547-570.

37 Ellen Mastenbroek, »EU Compliance: Still a «Black Hole»?», Journal of European Public Policy, 12, n. ${ }^{\circ} 6$ (2005): 1103-1120.

${ }^{38}$ Bernard Steunenberg y Michael Kaeding, «As time goes by: explaining the transposition of maritime directives», European Journal of Political Research, 48, n. 3 (2009): 432454.

39 Kaeding, «Determinants of Transpostition delay in The European Union»

40 Robert Thomson, René Torenvlied y Javier Arregui, «The Paradox of Compliance: Infrengement and delays in transposing European Union directives», British Journal of Political Science, 37, n. ${ }^{\circ} 4$ (2007): 685-709. 
fase del proceso de implementación. En este sentido, muchos autores señalan que las preferencias políticas de los EM deben ser incluidas como un factor explicativo. El ajuste de las preferencias, que habitualmente se refiere a la medida en que la preferencia de un EM difiere de la decisión final adoptada es la operacionalización que más se ha utilizado ${ }^{41}$. El argumento aquí implica que los gobiernos de los EM y sus respectivas administraciones pueden estar motivados a proteger los legados de las políticas existentes, siendo, por tanto, reticentes a implementar las políticas que se adoptan en Bruselas, sobre todo estas que requieran cambios profundos en el status quo doméstico ${ }^{42}$.

\section{Datos Empíricos sobre el nivel de conformidad de los EM con la legislación de la UE}

Esta sección muestra datos empíricos sobre el proceso de implementación de los diversos EM a lo largo del tiempo. Desagregamos los datos por tipos de infracción, por EM y en el caso de España también por sectores políticos en los que se producen las infracciones. Todos estos datos se han recogido de los informes anuales que la Comisión Europea realiza sobre la actividad legislativa. En algunos casos disponemos de datos e información desde 1986 mientras que en otros casos, los datos se circunscriben al periodo 1995-2015.

De acuerdo con los datos de la Comisión Europea para el periodo 19862010 , la mayor parte de los procedimientos de infracción no pasan de la primera fase (carta de notificación), esto es, los EM resuelven mayoritariamente en un estadio inicial los requerimientos de la Comisión Europea. Más específicamente, y de acuerdo con los informes anuales de la Comisión, para el periodo 1986-2015, el 67,46\% de los procedimientos de infracción abiertos no pasaron más allá de una carta de notificación, en el 24,9\% de los casos, la Comisión llegó a emitir un dictamen motivado y solamente en un 7,6\% del total de los casos, la Comisión llevó ante el TJUE los procedimientos abiertos de infracción. Por tanto, y de acuerdo a estos datos, tan sólo en un número reducido de casos, los EM se resisten a implementar correctamente la legislación europea.

41 Thomson, Torenvlied y Arregui, «The Paradox of Compliance: Infrengement and delays in transposing European Union directives»; Thomas König y Lars Mäder, «Nonconformable, partial and conformable transposition: A competing risk analysis of the transposition process of directives in the EU15», European Union Politics, 14, n. ${ }^{\circ} 1$ (2013): 46-69.

42 Thomson, Torenvlied y Arregui, «The Paradox of Compliance» 
El gráfico 1 muestra el número de infracciones abiertas por la Comisión Europea a los 15 y/o 27 EM en el periodo 1995-2015. Lo primero que se puede observar es que, en línea con los datos anteriores, existe una gran diferencia entre los procedimientos de infracción abiertos por la Comisión Europea y las infracciones finalmente declaradas por el TJUE. Por tanto, la inmensa mayoría de las infracciones se resuelven con éxito antes de que lleguen al TJUE, y el número de casos en los que se producen incumplimientos reiterados (y, por tanto, infracciones declaradas) son muy limitados. Los estados a los que la Comisión les ha abierto más procedimientos de infracción son Italia, Grecia y Francia. Estos países son también los estados con más infracciones declaradas por parte del TJUE. Por tanto, hay una clara relación entre infracciones abiertas e infracciones declaradas. Por otro lado, los países con menos infracciones abiertas son algunos países de Europa del Este, pero estas infracciones están claramente distorsionadas por su tardía entrada en la UE en el año 2004 (así como por el marco temporal de nuestro análisis). Entre los países anteriores al proceso de ampliación de la UE, en 2004, los países que menos infracciones declaradas tienen son Dinamarca y Suecia (ver gráfico 1).

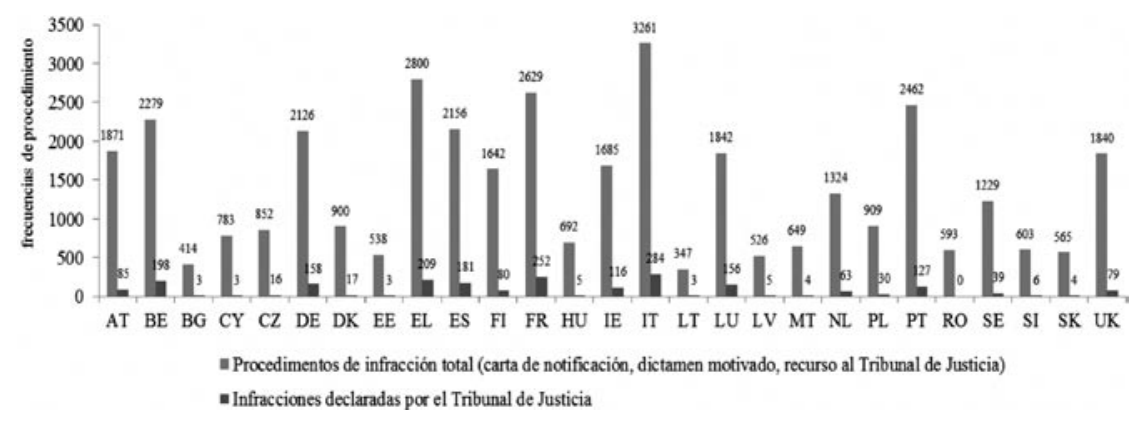

\section{Gráfico 1}

Comparación entre procedimientos de infracción abiertos e infracciones declaradas (1995-2015)

El gráfico 2, muestra los procedimientos de infracción abiertos por el TJUE a los EM. En este gráfico se puede observar con más nitidez los EM que tienen un mejor y un peor desempeño en la implementación de la legislación europea. Teniendo únicamente en cuenta los EM de la UE-15 (antes de las ampliaciones del 2004 y del 2007), el orden de los EM de mejor a peor desempeño en la implementación de la legislación europea es el siguiente: Dinamarca, Suecia, Holanda, Finlandia, Irlanda, Reino Unido, Luxemburgo, Austria, Alemania, España, Bélgica, Portugal, Francia, Gre- 
cia e Italia ${ }^{43}$ (ver gráfico 2). Entre los países de Europa del Este, el orden de los EM de mejor a peor desempeño en el proceso de implementación es el siguiente: Lituania, Bulgaria, Letonia, Estonia, Eslovaquia, Rumania, Eslovenia, Malta, Hungría, Chipre, República Checa y Polonia.

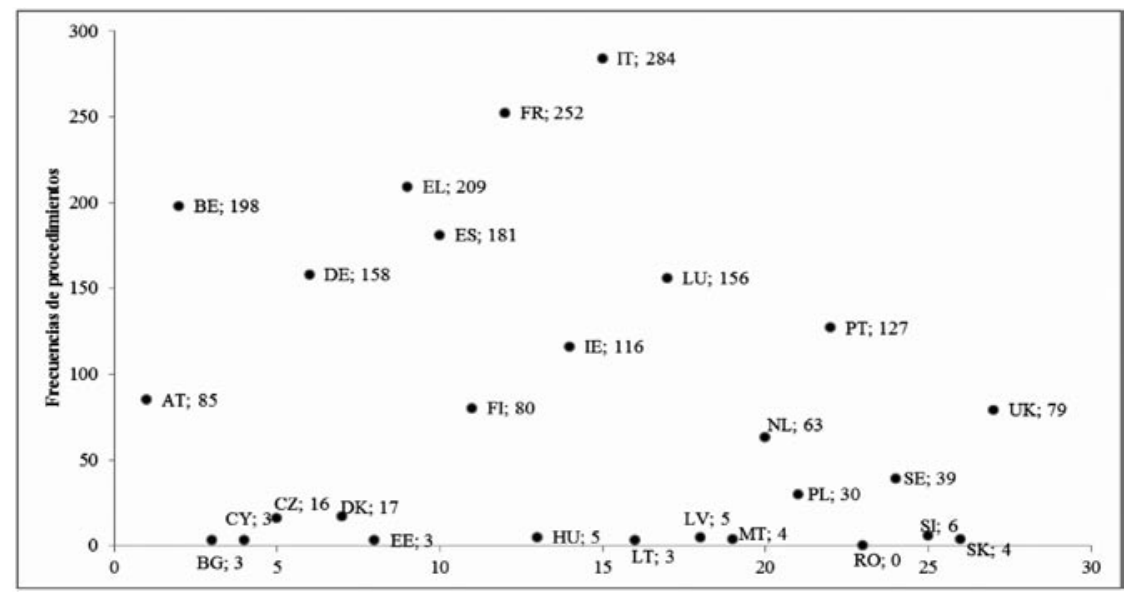

\section{Gráfico 2}

Total de infracciones declaradas por la CEJ (1995-2015)

También existe una importante variación en los procedimientos de infracción abiertos entre las distintas áreas políticas. En este caso, me voy

${ }^{43}$ Estos datos se corresponden en gran medida (aunque no son exactamente los mismos) con los cuatro modelos de implementación que han identificado Falkner et al. (2005) y Falkner y Treib (2008). Un primer modelo estaría formado por los países nórdicos en los que predomina la presencia de una cultura del respeto por el imperio de la ley tanto entre los actores políticos como entre los actores administrativos. Un segundo modelo estaría formado por Grecia, Portugal o Francia en el que la inexistencia de una cultura política y administrativa de cumplimiento con la ley llevaría a largos periodos de inercia burocrática que produciría problemas en el timing y en la cualidad de la implementación. En el tercer modelo la administración funciona relativamente bien, pero, sin embargo, los actores políticos no tienen interiorizado un sistema de cumplimiento con la ley. En este contexto, una correcta implementación dependerá de factores relacionados más con la agencia (las preferencias) de los actores que han de implementar dichas leyes. Los estados que Falkner et al. (2005) incluyen en este grupo son Bélgica, España, Alemania, Reino Unido y Holanda. Finalmente, los países del este de Europa formarían un cuarto grupo de estados en los que los procesos de adaptación a la legislación europea se caracterizan por ser procesos altamente politizados que muestran importantes limitaciones sobre todo en lo relativo a la implementación real de las leyes (Falkner y Treib 2008). 
a centrar en el caso de España (ver Gráfico 3). Los procedimientos de infracción abiertos a España en el periodo 1995-2015 por áreas políticas son los siguientes: 853 procedimientos de infracción en políticas medioambientales, 490 procedimientos de infracción en Mercado Interno, 200 infracciones en Energía, Transporte y Movilidad, 171 infracciones en Sanidad y Consumo, 147 infracciones en Impuestos y Aduanas, 142 infracciones en Agricultura y Desarrollo Rural, 98 casos en empleo y asuntos sociales y 86 infracciones en Innovación e Industria. En el resto de áreas políticas en número de infracciones abiertas es menos relevante: 68 en Justicia y Asuntos Internos, 44 en Competición, 23 en Información y Medios de Comunicación, 18 en Pesca y 6 procedimientos de infracción en asuntos económicos y financieros. De acuerdo con estos datos, España cumple la tendencia generalizada en la UE donde las dos áreas políticas que peor comportamiento muestran son las áreas relacionadas con políticas medioambientales, así como la implementación de políticas sobre el mercado interno.

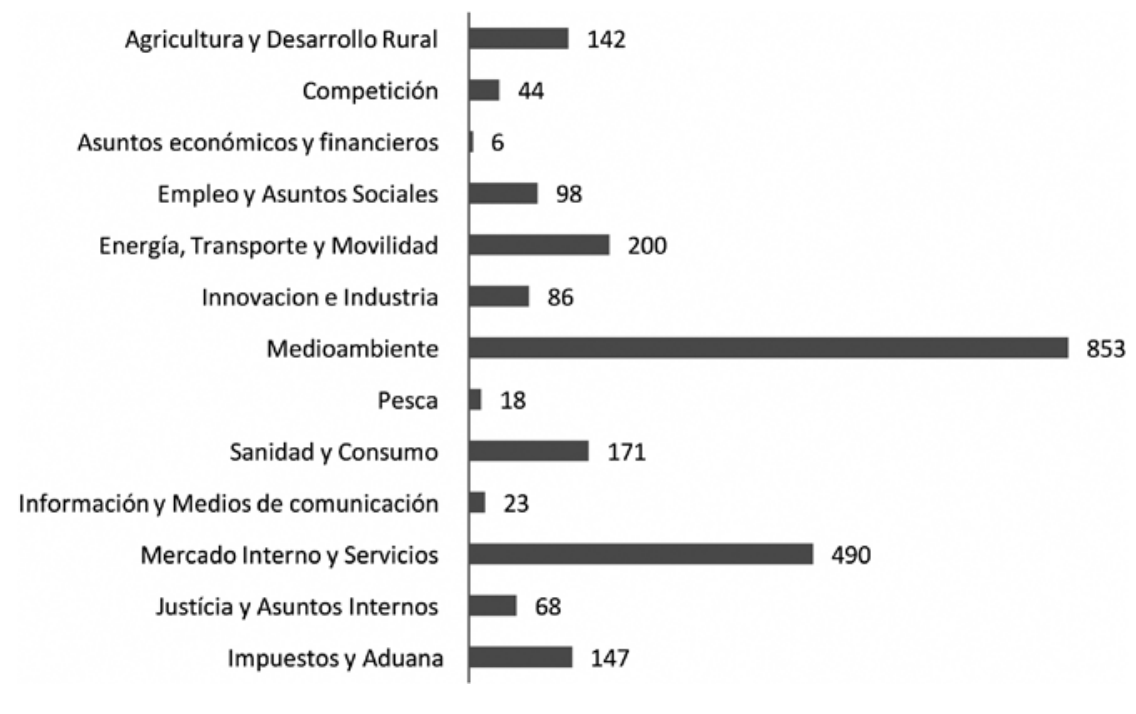

Gráfico 3

Procedimientos de infracción abiertos a España por Área Política (1995-2015)

A la hora de explicar estos casos de infracción por parte de los EM, no es infrecuente encontrar en la literatura «hallazgos» contradictorios en relación al peso explicativo que tienen las distintas variables utilizadas. 
Sin embargo, también existe cierto consenso sobre algunos factores que tienen un peso importante cuando se intenta explicar el cumplimiento o incumplimiento de los EM con la legislación europea.

Por ejemplo, existe consenso en la literatura de que la política doméstica es un factor determinante a la hora de originar una correcta implementación de la legislación europea ${ }^{44}$. También existe evidencia de que las preferencias de los partidos que están en el gobierno de los EM en el momento de transponer una ley son un factor determinante para una correcta implementación ${ }^{45}$. Por otro lado, muchos estudios corroboran la importancia de las capacidades administrativas ${ }^{46}$. En este sentido, las burocracias más eficaces llevan a cabo el proceso de transposición e implementación de una manera más rápida y efectiva ${ }^{47}$.

También existe evidencia de que más allá de la simple eficacia de la burocracia, el federalismo (y una de sus consecuencias: los problemas de coordinación entre actores) y un mayor número de jugadores con capacidad de veto $^{48}$ desempeñan un impacto negativo ${ }^{49}$. Diversos estudios también muestran la relación entre el proceso de implementación y el proceso de negociación. Thomson et al. (2007) muestran que los EM que se oponen a una Directiva durante la negociación en el Consejo tienen una mayor probabilidad de que incumplan con dicha ley. En la misma línea, un número de investigaciones han encontrado que cuanto mayor es la discreción que la Comisión Europea otorga a los EM a la hora implementar una Directiva, mayor éxito en la transposición e implementación de dicha Di-

${ }^{44}$ Gerda Falkner y Oliver Treib, «Three worlds of compliance or four? The EU-15 compared to new member states», Journal of Common Market Studies, 46, n. ${ }^{\circ} 2$ (2008): 293-313; Toshkov, «Taking Stock: A review of quantitative studies»; Börzel et al., «Obstinate and Inefficient: Why Member States do not comply with European law»

45 Andreas J. Obermaier, The End of Territoriality? The Impact of ECJ Rulings on British, German and French Social Policy, (Londres: Ashgate, 2009); Michael Blauberger, «National Responses to European Court Jurisprudence», West European Politics, 37, n. 3 (2014): 457-474.

${ }^{46}$ Heather A. D. Mbaye, «Why National States Comply with Supranational Law»; Kaeding, «Determinants of Transpostition delay in The European Union»; Markus Haverland y Marleen Romeijn, «Do member states make European policies work? Analysing the EU transposition deficit», Public Administration, 85, n. 3 (2007): 757-778; Berglund, Putting Politics into Perspective; Börzel et al., «Obstinate and Inefficient: Why Member States do not comply with European law»

47 Berglund, Putting Politics into Perspective: A study on the Implementation of EU Public Utilities Directives; Börzel et al., «Obstinate and Inefficient»; Perkins and Neumayer, «Do membership benefits by regulatory compliance?»

48 Tsebelis, Veto Players: How Political Institutions Work

49 Toshkov, «Taking Stock: A review of quantitative studies of transposition and Implementation of EU Law» 
rectiva ${ }^{50}$. Esto es así, porque la discreción otorga a los EM un margen mayor para que estos puedan transponer la legislación europea teniendo en cuenta sus circunstancias domésticas. Por tanto, a mayor discreción mejor transposición ${ }^{51}$.

A partir de estos consensos que ha generado la literatura, es obvio que diferentes variables o factores explicativos pueden ser relevantes a la hora de explicar el proceso de transposición y/o implementación de un país o de un sector político en un determinado momento, pero pueden ser menos relevantes para explicar los mismos procesos en otro EM o en otras áreas políticas. De hecho, para poder tener un mapa completo de los procesos de implementación de leyes europeas y de sus factores explicativos precisaríamos contextualizar las 28 tradiciones europeas sobre los procesos de implementación legislativa. Algo que todavía no se ha llevado a término.

\section{IV. ¿Existe en los EM un problema de conformidad con la legislación de la UE?}

Al analizar la evidencia empírica nos encontramos ante una realidad múltiple y compleja, en la que existe una gran variación en los niveles de cumplimiento de la legislación europea entre EM, así como entre diferentes áreas políticas. A esto hay que añadir que el proceso de implementación de la legislación europea está rodeado de una extrema complejidad burocrática. Esto es así porque la Comisión Europea (actor encargado de supervisar la implementación correcta de la legislación europea) no dispone de los recursos y mecanismos de control suficientes para asegurar una adecuada aplicación de toda la legislación vinculante (Directivas, Reglamentos y Decisiones) que se adopta en Bruselas. Este hecho se ve agravado, en no pocas ocasiones, por la falta de iniciativa e incluso de colaboración por parte de los $\mathrm{EM}^{52}$.

¿Existe en la UE un problema de cumplimiento con la legislación europea? Estrictamente hablando, no se disponen de datos objetivos con

50 Thomson, Torenvlied y Arregui, «The Paradox of Compliance»; Bernard Steunenberg y Dimiter Toshkov, «Comparing Transposition in the 27 member states of the EU: the impact of discretion and legal fit», Journal of European public Policy, 16, n. 7 (2009): 951-970.

51 Thomson, Torenvlied y Arregui, «The Paradox of Compliance»; Asya Zhelyazkova y Rene Torenvlied, «The successful transposition of European provision by member states: application to the Framework Equality Directive», Journal of European Public Policy, 18, n. ${ }^{\circ} 5$ (2011): 690-708.

52 Börzel, «Non-compliance in the European Union: pathology or statistical artefact» 
los que poder contestar esta pregunta. Los procedimientos de infracción abiertos por la Comisión no son indicadores reales y objetivos sobre el incumplimiento de la legislación europea por parte de los EM. Los procedimientos de infracción tan sólo pueden servir como indicadores relativos sobre dicho incumplimiento. Esto es así porque no está claro que ni la Comisión ni siquiera los propios EM ${ }^{53}$ dispongan de datos en los que se pueda confiar sobre el éxito en la implementación de la legislación europea. Sin embargo, este artículo de investigación ha confiado su análisis en los únicos datos oficiales que existen de la Comisión Europea y del TJUE sobre problemas identificados en los EM con la legislación europea.

Además, hay que tener presente que algunos estudios han puesto de manifiesto que la acción de la Comisión Europea no es siempre una acción neutral. Thomson et al. (2007) han mostrado que en estos casos en los que el Consejo y el Parlamento adoptan una decisión que va en contra de las preferencias de la Comisión Europea, es más probable que a un estado se le abra un procedimiento de infracción (por parte de la propia Comisión). Por otro lado, König y Mäder ${ }^{54}$ han demostrado algo similar en relación a la variable salience (o intensidad de las preferencias) de la Comisión en relación a una determinada ley: cuanto mayor es la intensidad de las preferencias de la Comisión en una ley, mayor es la probabilidad de que ésta abra un procedimiento de infracción a un EM. Por tanto, existe evidencia empírica sobre la utilización estratégica que puede hacer la Comisión a la hora de abrir procedimientos de infracción.

Algunos estudios han mostrado que debido a la falta de recursos, la Comisión Europea tan sólo puede detectar infracciones de los EM en algunos casos, mientras que en muchos otros casos en los que la trasposición de una Directiva no se ha realizado de un modo correcto, la Comisión no siempre tiene mecanismos para identificar los problemas. De hecho hay autores ${ }^{55}$ que argumentan que los datos de infracciones de que dispone la Comisión Europea tan sólo son «la punta del iceberg» de los casos de infracción que

${ }^{53}$ Por poner un ejemplo, los EM no tienen datos sobre el proceso de implementación real de la Directiva Antidiscriminación en el trabajo por motivos de raza, orientación sexual o religión, y si los EM no tienen datos, la Comisión Europea todavía está más huérfana de datos objetivos y de instrumentos adecuados para poder estimar el alcance del posible in(cumplimiento) de los EM.

54 Thomas König y Lars Mäder, « The Strategic Nature of Compliance: An Empirical Evaluation of Law Implementation in the Central Monitoring System of the European Union», American Journal of Political Science, $58 \mathrm{n} .^{\circ} 1$ (2014): 246-263.

55 Ver por ejemplo: Gerda Falkner et al, Complying with Europe: EU Harmonisation and Soft Law in the Member States (Cambridge: Cambridge University Press, 2005), 204; König y Mäder, « The Strategic Nature of Compliance» 
existen en el mundo real. A pesar de todo ello, no existe evidencia alguna de que la UE tenga un serio problema de incumplimiento con su legislación o haya sobrepasado el umbral que haga difícil el funcionamiento del mercado único ${ }^{56}$.

\section{Conclusiones}

Este artículo ha mostrado que existe una variación importante en el desempeño de los distintos EM en relación al proceso de transposición e implementación de la legislación europea. Los países nórdicos junto con Holanda, Irlanda y Reino Unido son los que mejor desempeño ofrecen en los procesos de transposición e implementación de acuerdo con las estadísticas oficiales, les siguen países como Luxemburgo, Austria, Alemania o España. Un tercer grupo de estados entre los que se encuentran los que peor desempeño presentan en los procesos de transposición e implementación son Italia, Francia, Bélgica o Grecia. Los países del Este de Europa también han sido estudiados de un modo sistemático. Los países del Este que muestran mejor desempeño son los países Bálticos y el país que tiene más problemas a la hora de implementar la legislación europea es Polonia. Sin embargo, en el análisis sobre los países de Europea del Este, posiblemente, no existe una referencia temporal suficientemente amplia para un análisis consistente. Sin embargo, tal y como muestran los datos analizados, algunos de estos países muestran limitaciones importantes en la gestión y ejecución en los proceso de implementación ${ }^{57}$.

La variación en el desempeño de la implementación legislativa entre los distintos EM se ha explicado de acuerdo a una serie de factores positivos (que facilitan una correcta implementación) y factores negativos (que dificultan dicha implementación). En líneas generales, la efectividad administrativa, el control parlamentario y la capacidad en los procesos de coordinación entre actores tienen una influencia positiva, mientras que el federalismo, la ineficacia administrativa, el número de jugadores veto y la existencia de posibles conflictos (de preferencias) entre actores tienen un impacto negativo a la hora de cumplir con la legislación europea.

La variación en los procesos de implementación no sólo se da entre EM sino que también tiene lugar entre sectores políticos. En este sentido, en el caso de España, los mayores problemas en el proceso de implementación se producen en el área de políticas medioambientales y también en políticas

56 Börzel, «Non-compliance in the European Union».

57 Falkner y Treib, «Three worlds of compliance or four?» 
del mercado interno. El estudio de Haverland et al. 2011, va en la misma dirección cuando muestra las múltiples problemas a la hora de implementar las políticas de mercado interno en distintas áreas (telecomunicaciones, sanidad, energía, etc.). Los problemas de implementación en temas medioambientales tienen que ver, posiblemente, con el hecho de que es el área en la que existe más legislación en la UE. Estos problemas en áreas políticas tan importantes se pueden explicar por el hecho de que la Comisión no tiene técnicos trabajando en los EM cuyo objetivo sea identificar incumplimientos de la legislación europea ${ }^{58}$, así como capacidad suficiente para hacer cumplir su legislación En la mayoría de los casos, depende de terceros actores que le puedan notificar tales incumplimientos. En temas medioambientales, las organizaciones no gubernamentales son actores muy activos a la hora de informar sobre incumplimientos, mientras que en otras áreas políticas la colaboración de terceros actores es menor. En relación al mercado interno, también se producen más denuncias de actores privados (que en otras áreas políticas) cuando estos ven amenazados sus intereses por una implementación deficiente de la legislación europea ${ }^{59}$.

Para poder explicar mejor el cumplimiento de la ley europea por parte de los EM, posiblemente, deberíamos incorporar el poder normativo de la ley en nuestras explicaciones ${ }^{60}$, sin perder de vista el poder de las explicaciones instrumentales y de gestión. En realidad para entender el cumplimiento de la ley europea por parte de los EM necesitamos una especie de síntesis teórica que tienda puentes entre distintos tipos de variables y teorías, y que pueda incorporar factores normativos, instrumentales, de capacidades, etc. Hasta la fecha no se ha trabajado en esta dirección y esta es posiblemente la dirección más productiva, sobre todo, a la hora de poder especificar las condiciones en las que es más probable que se produzcan políticas de conformidad con la legislación europea.

58 La única excepción desde mediados de los años 90 son las agencias reguladoras, que desempeñan un importante papel a la hora de identificar problemas en el proceso de implementación. Majone (2000: 290) define las agencias como una variedad de organizaciones que tienen funciones de naturaleza gubernamental y que al mismo tiempo llevan a cabo su actividad al margen de la estructura del gobierno. Actualmente existen en la UE alrededor de unas 30 agencias reguladoras y cada vez tienen un papel más relevante en la supervisión de los procesos de implementación legislativa, sobre todo en temas importantes en el desarrollo del mercado único. Por ejemplo, Bourblanc et al. (2013) señalan la importancia de este tipo de agencias a través de un estudio realizado sobre la implementación en varios EM sobre la Directiva Marco del Agua. Este tipo de hallazgos muestran la importancia que tiene o puede adquirir las teorías top-down a la hora de explicar el éxito en la implementación de la legislación europea en los EM.

59 Börzel, «Non-compliance in the European Union».

${ }^{60}$ Derek Beach, «Why Governments Comply». 
Este artículo también ha mostrado que no existe suficiente evidencia empírica de que el incumplimiento de los EM en relación con la legislación europea sea un problema de primer orden para el proceso de integración. De hecho, los umbrales de cumplimiento con la legislación de la UE por parte de los EM se sitúa en unos parámetros razonables, de acuerdo con las estadísticas oficiales: tan sólo un 7,6\% de los procedimientos de infracción abiertos por la Comisión acaban en el TJUE. Esto significa que los EM son capaces de solucionar en el tiempo una mayoría de casos que la Comisión identifica como problemáticos. Sin embargo, es obvio que los datos oficiales no cubren todos los problemas de implementación legislativa en la UE. Esto no significa que exista un problema sustancial en el proceso de la implementación legislativa en la UE, todavía más si tenemos en cuenta la miles de Directivas, Reglamentos, Decisiones y artículos de los Tratados que forman parte del acervo legislativo comunitario ${ }^{61}$.

Finalmente, las investigaciones sobre implementación práctica de las leyes europeas en los EM está todavía ausente en la literatura (si exceptuamos algunos estudios de caso). Por tanto, en términos prácticos el proceso de implementación real (no legislativa) en los estados de la EU sigue siendo una caja negra.

\section{Agradecimientos}

Agradezco a los revisores anónimos de este artículo por sus comentarios que han mejorado la versión previa de este artículo. También agradezco el apoyo recibido por el Ministerio de Economía y Competitividad (Proyecto CSO2012-35554) que ha posibilitado poder llevar a cabo esta investigación.

\section{Referencias bibliográficas}

Angelova, Mariyana, Tanja Dannwolf y Thomas König. «How Robust are Compliance Findings? A Research Synthesis», Journal of European Public Policy, n. 19 (2012): 1269-1291.

Bardach, Eugene. The Implementation Game: What Happens after a Bill Becomes a Law. Cambridge: MIT Press. 1977.

Beach, Derek. «Why Governments Comply: An Integrative Compliance Model that Bridges the Gap Between Instrumental and Normative Models of Compliance», Journal of European Public Policy, n. ${ }^{\circ} 12$ (2005): 113-142.

${ }^{61}$ Simon Hix y Bjorn Hoyland, The Political System of the European Union (New York: Palgrave Macmillan, 2011) 
Berglund, Sara. Putting Politics into Perspective: A Study on the Implementation of EU Public Utilities Directives. Delft: Eburon. 2009.

Blauberger, Michael. «Compliance with Rules of Negative Integration: European State Aid Control in the New Member States», Journal of European Public Policy, n. ${ }^{\circ} 16$ (2009):1030-1046.

Blauberger, Michael. «National Responses to European Court Jurisprudence», West European Politics, n. ${ }^{\circ}$ 37(2014): 457-474.

Börzel, Tanja A.. «Non-compliance in the European Union: pathology or statistical artifact», Journal of European Public Policy, n. ${ }^{\circ} 8$ (2001): 803-24.

Börzel, Tanja A.. «Participation Through Law Enforcement: The Case of the European Union», Comparative Political Studies, n. ${ }^{\circ}$ 39(2006): 128-152.

Börzel Tanja A., Tobias Hofmann, Diana Panke, y Carina Sprungk. «Obstinate and Inefficient: Why Member States do not comply with European law», Comparative Political Studies, n. ${ }^{\circ} 43$ (2010): 1363-90.

Bourblanc, Magalie, Ann Crabbe, Duncan Liefferink y Mark Wiering. «The Marathon of the Hare and the Tortoise: Implementing the EU Water Framework Directive», Journal of Environmental Planning and Management, n. ${ }^{\circ} 56$ (2013): 1449-1467.

Chayes, Abram y Antonia Handler Chayes. The New Sovereignty. Compliance with International Regulatory Agreements. Cambridge: Harvard University Press, 1995.

Chayes, Abram, Antonia Handler Chayes y Ronald B. Mitchell. «Managing Compliance: A Comparative Perspective». En Engaging Countries: Strengthning Compliance with International Environmental Accords, editado por E.B. Weiss y H.K., Jakobsen, 39-62. Cambridge: MIT Press, 1998.

Ciavarini Azzi, Guiseppe. L'application du droit communautaire par les etats membres. Maastrich: European Institute of Public Administration, 1988.

Downs, George W., David M. Rocke y Peter N. Barsoom, «Is the Good News about Compliance also Good News about Cooperation?», International Organization, n. 50 (1996): 379-406.

Duina, Francesco G. «Explaining Legal Implementation in the European Union», International Journal of the Sociology of Law, n. 25 (1997): 155-179.

Evans, Peter. Embedded Autonomy: States and Industrial Transformations. Princeton: Princeton University Press, 1999.

Falkner, Gerda, Oliver Treib, Miriam Hartlapp y Simone Leiber. Complying with Europe: EU Harmonisation and Soft Law in the Member States. Cambridge: Cambridge University Press, 2005.

Falkner, Gerda. y Oliver Treib. «Three worlds of compliance or four? The EU-15 compared to new member states», Journal of Common Market Studies, n. ${ }^{\circ} 46$ (1998): 293-313.

Garret, Geofrrey. «The Politics of legal integration in the European Union», International Organization, n. ${ }^{\circ} 49$ (1995): 171-181.

Giuliani, Marco. «Europeanization in Comparative Perspective: Institutional Fit and National Adaptation». En The Politics of Europeanization, editado por Kevin Featherstone y Claudio M. Radaelli. Oxford: Oxford University Press, 2003.

Haverland, Markus. «National Adaptation to European Integration: The Importance of Institutional Veto Points», Journal of Public Policy, n. 20 (2000): 83-103. 
Haverland, Markus y Marleen Romeijn. «Do member states make European policies work? Analysing the EU transposition deficit», Public Administration, n. ${ }^{\circ} 85$ (2007): 757-78.

Haverland, Markus, Bernard Steunenberg y Frans Van Waarden. «Sectors at Different Speeds: Analyzing Transpositions Deficits in the European Union», Journal of Common Market Studies, n. ${ }^{\circ} 49$ (2011): 265-291.

Hix, Simon., y Bjorn Hoyland.. The Political System of the European Union. New York: Palgrave Macmillan, 2011.

Kaeding, Michael. «Determinants of Transposition Delay in the European Union», Journal of Public Policy, n. 26 (2006): 229-253.

Linos, Katerina. «How can International Organizations Shape National Welfare States? Evidence from Compliance with European Union directives», Comparative Political Studies, n. 40 (2007): 547-70.

Keohane, Robert. y Joseph Nye. Power and Interdependence. Boston: Little, Brown and Company, 1977.

König, Thomas y Lars Mäder. «Non-conformable, Partial and Conformable Transposition: A Competing Risk Analysis of the Transposition Process of Directives in the EU15», European Union Politics, n. ${ }^{\circ} 14$ (2013): 46-69.

König, Thomas y Lars Mäder. «The Strategic Nature of Compliance: An Empirical Evaluation of Law Implementation in the Central Monitoring System of the European Union», American Journal of Political Science, n. ${ }^{\circ} 58$ (2014): 246-263.

Majone, Giandomenico. «The Credibility Crisis of Community Regulation», Journal of Common Market Studies, n. ${ }^{\circ} 38$ (2000): 273-302.

Martin, Lisa L. Coercive Cooperation: Explaining Multilateral Economic Sanctions. Princeton: Princeton University Press, 1992.

Mastenbroek, Ellen. «EU Compliance: Still a "Black Hole”?», Journal of European Public Policy, n. ${ }^{\circ} 12$ (2005): 1103-1120.

Mbaye, Heather A. D.. «Why National States Comply with Supranational Law: Explaining Implementation Infringements in the European Union 1972-1993», European Union Politics, n. ${ }^{\circ} 2$ (2001): 259-281.

Obermaier, Andreas J.. «The National Judiciary - Sword of European Court of Justice Rulings: The Example of the Kohll/Decker Jurisprudence», European Law Journal, n. 14 (2008): 735-752.

Obermaier, Andreas J.. The End of Territoriality? The Impact of ECJ Rulings on British, German and French Social Policy. Londres: Ashgate, 2009.

Perkins, Richard y Eric Neumayer. «Do membership benefits by regulatory compliance? An empirical analysis of EU Directives 1978-99», European Union Politics, n. ${ }^{\circ} 8$ (2007): 180-206.

Pressman, Jeffrey L. y Aaron Wildavsky. Implementation: How Great Expectations in Washington Are Dashed in Oakland; Or, Why It's Amazing that Federal Programs Work at All. Berkeley: University of California Press, 1973.

Sabatier, Paul A. y Daniel A. Mazmanian. «The Implementation of Public Policy: A Framework of Analysis». En Effective Policy Implementation, editado por Paul A. Sabatier y Daniel A. Mazmanian. 3-35. Lexington: Lexington Books, 1981.

Siedentopf, Heinrich y Jacques Ziller. Making European Policies Work: The Implementation of Community Legislation in the Member States. Londres: Sage, 1988. 
Siegel, Scott Nicholas. The Political Economy of Noncompliance: Adjusting to the Single European Market. Abingdon: Routledge, 2011.

Steunenberg, Bernard y Michael Kaeding. "As time goes by: explaining the transposition of maritime directives», European Journal of Political Research, n. ${ }^{\circ} 48$ (2009): 432-54.

Steunenberg, Bernard y Dimiter Toshkov. «Comparing transposition in the 27 member states of the EU: the impact of discretion and legal fit», Journal of European Public Policy, n. ${ }^{\circ} 16$ (2009): 951-70.

Tallberg, Jonas. «Paths to Compliance: Enforcement, Management, and the European Union». International Organization, n. 56 (2002): 609-643.

Thomson, Robert, Frans N. Stokman, Thomas König y Cristopher H. Achen. The European Union Decides: Testing Theories of European Decision Making. Cambridge: Cambridge University Press, 2006.

Thomson, Robert, René Torenvlied y Javier Arregui. «The Paradox of Compliance: Infringements and delays in transposing European Union directives», British Journal of Political Science, n. 37 (2007): 685-709.

Toshkov, Dimiter. «Taking Stock: A Review of Quantitative Studies of Transposition and Implementation of EU Law», Institute for European Integration Research, EIF Working Paper, n. ${ }^{\circ} 1$ (2010)

Tsebelis, George. Veto Players: How Political Institutions Work. Princeton: Princeton University Press, 2002.

Treib, Oliver. «Implementing and Complying with EU Governance Outputs», Living Reviews in European Governance, n. ${ }^{\circ} 9$ (2014):1-47.

Versluis, Esther. «Explaining Variations in Implementation of EU Directives», European Integration online Papers, $\mathrm{n}^{\circ} 8$ (2004).

Zhelyazkova, Asya, y René Torenvlied. «The successful transposition of European Provisions by member states: application to the Framework Equality Directive», Journal of European Public Policy, n. ${ }^{\circ} 18$ (2011): 690-708. 


\section{Derechos de autor (Copyright)}

Los derechos de autor (distribución, comunicación pública, reproducción e inclusión en bases de datos de indexación y repositorios institucionales) de esta publicación pertenecen a la editorial Universidad de Deusto. El acceso al contenido digital de cualquier número de Cuadernos Europeos de Deusto (CED) es gratuito, transcurridos 6 meses desde su publicación. Los trabajos podrán descargarse, copiar y difundir, sin fines comerciales y según lo previsto por la ley. Así mismo, los trabajos editados en CED pueden ser publicados con posterioridad en otros medios o revistas, siempre que el autor indique con claridad y en la primera nota a pie de página que el trabajo se publicó por primera vez en CED, con indicación del número, año, páginas y DOI (si procede). 